\title{
Applying a correction factor to gated blood pool SPECT left ejection fraction value: Can this approach be used to get around a longstanding hurdle?
}

\author{
Sylvain Prévost, $M D,{ }^{a}$ and Etienne Rousseau, MD, B. Eng. ${ }^{a}$ \\ a Department of Nuclear Medicine and Radiobiology, Centre de recherche CHUS, Universite de \\ Sherbrooke, Sherbrooke, Canada
}

Received Feb 19, 2019; accepted Feb 19, 2019

doi: $10.1007 / \mathrm{s} 12350-019-01668-1$

\section{See related article, pp. 1539-1549}

It is somewhat surprising, and even disappointing to some extent, that gated blood pool SPECT still cannot be used routinely to determine left ventricular ejection fraction nowadays. Even though SPECT provides some clear advantages over planar imaging such as reducing ventricle and atrium overlap, background related variability, and even intra- and inter-observer variability according to some authors, ${ }^{1,2}$ its role remains limited in day-to-day clinical practice being only used to qualitatively assess ventricular wall motion in most nuclear medicine departments.

Among the factors that may have precluded a more widespread use is the relatively important discordance sometimes observed between ejection fraction values obtained using SPECT acquisitions versus traditional planar acquisitions, which have been accepted as the gold standard for decades, even though many publications have repeatedly reported acceptable correlations between the two methods. ${ }^{3,4}$ This discordance is usually more obvious in patients with an ejection fraction in the normal range such as those referred for cardiac pharmacotoxicity screening which accounts for most indications in many imaging departments.

In this issue of the Journal of Nuclear Cardiology, Tissot et al demonstrate clearly the feasibility of

\footnotetext{
Reprint requests: Sylvain Prévost, MD, Department of Nuclear Medicine and Radiobiology, Centre de recherche CHUS, Universite de Sherbrooke, Sherbrooke, Canada; sylvain.prevost@usherbrooke.ca J Nucl Cardiol 2019;26:1550-1.

1071-3581/\$34.00

Copyright (C) 2019 American Society of Nuclear Cardiology.
}

applying a simple correction factor to compensate for the overestimation of gated blood pool SPECT ejection fraction values observed in their study. The procedure allows to obtain ejection fraction values closer to those obtained with planar imaging which could eventually be used interchangeably if the method turns out to be robust enough in the long run. It would thus allow to keep the so widely accepted, almost iconic figure of $50 \%$ for normal lower limit of left ventricular ejection fraction. Their method is relatively easy to implement, necessitating only a small set of patients with normal ejection fraction on 2D imaging as a reference.

As they suggest, SPECT only imaging performed on dedicated cardiac CZT cameras would allow to dramatically reduce injected activity and absorbed radiation dose by approximately $70 \%$. It could probably allow some significant dose reduction on conventional gamma cameras as well, although to a lesser extent, especially if data are reconstructed using resolution recovery reconstruction algorithms, as reported before for MPI studies. ${ }^{5}$

Alternatively, the method could also be useful for cases in which planar imaging provides doubtfully decreased or even evidently erroneous ejection fraction values as can be seen when there is an important overlap of left ventricle and atrium, with poor red-blood cell labeling or with increased background of any cause, whereas the SPECT value obtained for the same patient frequently appears normal. This is unfortunately not uncommon, now that most modern cameras do not offer the possibility to perform a caudal tilt of the detector, which allowed a better atrio-ventricular separation.

On the other hand, SPECT imaging has some inherent issues as well. It is especially affected by patient motion and region contouring is sometimes erroneous and can completely fail in some cases. Thus, 
one must pay close attention to all quality control in the process. Each commercially available cardiac software package has its own advantages and limitations. Particularly contour recognition performance might vary between vendors and might be dependent on reconstruction parameters. It would be crucial to keep those parameters constant once the correction factors are established; otherwise, the correction factors would have to be recalculated.

To our knowledge, it is the first time that an author proposes to apply a correction factor to compensate for the observed discordance between planar and SPECT ejection fraction values, even though overestimation of SPECT LVEF values versus planar has been documented for years ${ }^{4}$ One should note that the inverse situation has been reported as well, ${ }^{6}$ emphasizing that variability in acquisition parameters and type of equipment can translate into a wide range of possible outcome. While the proposed method might appear simple to solve such a complex problem, it can be tested at almost no cost and does not necessitate any additional software. It is certainly worth giving it an honest try, before the nuclear medicine community finds a more definitive standardized solution. It might not be the final step, but it could give some wings to a once promising technique which it seems to us has yet to reach its full potential.

\section{Disclosure}

Sylvain Prévost and Etienne Rousseau declare that they have nothing to disclose.

\section{References}

1. Jensen MM, Schmidt U, Huang C, Zerahn B. Gated tomographic radionuclide angiography using cadmium-zinc-telluride detector gamma camera; comparison to traditional gamma cameras. J Nucl Cardiol. 2014;21:384-96. https://doi.org/10.1007/s12350-013-9844-6.

2. Jensen MM, Haase C, Zerahn B. Interstudy repeatability of left and right ventricular volume estimations by serial-gated tomographic radionuclide angiographies using a cadmium-zinc-telluride detector gamma camera. Clin Physiol Funct Imaging. 2015;35:418-24. https:// doi.org/10.1111/cpf.12178.

3. Chen Y-C, Ko C-L, Yen R-F, et al. Comparison of biventricular ejection fractions using cadmium-zinc-telluride SPECT and planar equilibrium radionuclide angiography. J Nucl Cardiol. 2016;23:348-61. https://doi.org/10.1007/s12350-015-0367-1.

4. Bartlett ML, Srinivasan G, Barker WC, et al. Left ventricular ejection fraction: comparison of results from planar and SPECT gated blood-pool studies. J Nucl Med. 1996;37:1795-9.

5. Zafrir N, Solodky A, Ben-Shlomo A, et al. Feasibility of myocardial perfusion imaging with half the radiation dose using ordered-subset expectation maximization with resolution recovery software. J Nucl Cardiol. 2012;19:704-12. https://doi.org/10.1007/s12350-012-9552-7.

6. Pelletier-Galarneau M, Finnerty V, Tan S, et al. Assessment of left ventricular ejection fraction with cardiofocal collimators: Comparison between IQ-SPECT, planar equilibrium radionuclide angiography, and cardiac magnetic resonance. J Nucl Cardiol. 2018. https://doi.org/10.1007/s12350-018-1251-6.

Publisher's Note Springer Nature remains neutral with regard to jurisdictional claims in published maps and institutional affiliations. 